\title{
APPLICATION OF DICYANIN* TO THE PHOTOGRAPHY OF STELLAR SPECTRA
}

By Paul W. Merrill

\section{CONTENTS}

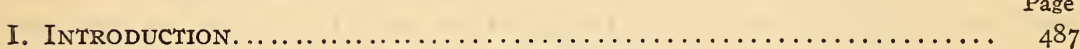

I. Advantages of photography in spectroscopy............. 487

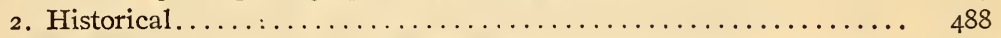

3. Purpose of this investigation....................... 490

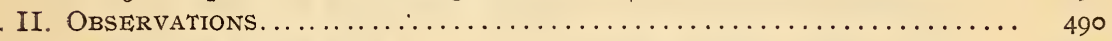

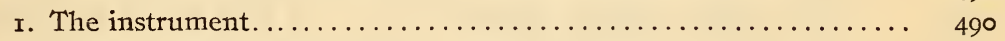

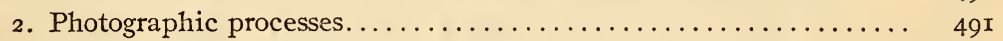

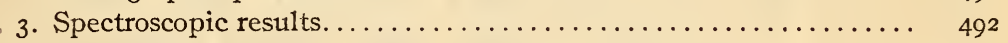

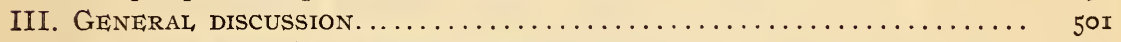

r. Identification of band at wave length $.760 \mu \ldots \ldots \ldots \ldots \ldots \ldots \ldots$ 50I

2. Distribution of energy in stellar spectra................. $5^{02}$

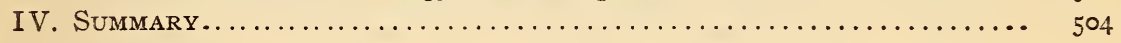

\section{INTRODUCTION}

\section{ADVANTAGES OF PHOTOGRAPHY IN SPECTROSCOPY}

It has become almost axiomatic in modern spectroscopy that photography should be applied wherever possible. This is particularly true in astrophysics, since the observational conditions give photography especially great advantages. It is a mistake to suppose that these advantages are limited to the more refrangible portions of the spectrum. In the spectral region to which the eye is most sensitive, namely, near wave length $.56 \mu$ (yellowgreen), a rather long exposure is required to show photographically the faintest details seen by a skillful observer, but even here it is only under special conditions that visual results are of great value as compared with a photographic record.

The visibility of radiation ${ }^{1}$ falls rapidly on either side of $\lambda_{5} 6,{ }^{2}$ being equal to I per cent of the maximum at $\lambda_{4}$ (violet) and at

* Dicyanin, a synthetic dye used for sensitizing photographic plates.

1 Coblentz and Emerson, this Bulletin, 14, p. 167; 1917.

2 Throughout this paper $\lambda$ will be used as an abbreviation for wave length. In referring to wave lengths between 0.3 and $1.0 \mu$ ( 3000 and 10000 angstroms) two or more significant figures will be given without decimal points or units. Thus, $\lambda_{5} 6$ is to be understood as wave length $0.56 \mu$ ( ${ }_{5} 600$ angstroms); $\lambda_{5} 60$ or $\lambda_{5} 600$ would refer to the same wave length, but would indicate greater accuracy. 
$\lambda 69$ (red). From $\lambda 69$ on toward longer waves this value is halved every roo angstroms, so that to produce a given sensation of vision 5000 times as much energy is required at $\lambda_{75}$ as at $\lambda_{5} 6$. The inefficiency of the eye for the red rays is, perhaps, not generally recognized, since it has not been emphasized so greatly as in the case of the violet rays by the success of photography.

To the eye a continuous spectrum is usually terminated at the red end, not by the lack of energy but by the ineffectiveness of the longer waves in producing vision.

This fact has been known, as far as the solar spectrum is concerned, since the days of the elder Herschel, and has for many years been inferred to hold true in stellar spectra from the assumption that the spectral-energy curve is comparable with that of a black body. Nichols's observations in 1900 showed the existence of considerable infra-red energy, at least for the star Arcturus. "The ratio greater than 2 to $\mathrm{I}$, of the total radiation of Arcturus to that of Vega (stars which by most observers are estimated to be of nearly equal photometric magnitude) indicates a proportionately more intense infra-red spectrum for the former than for the letter star."3 By observations of stars with and without a water screen Coblentz ${ }^{4}$ has recently proved that a large fraction of the energy in stellar spectra is of greater wave length than $\mathrm{I}_{4} 4 \mu$ ( 14000 angstroms).

The results obtained in the infra-red by the use of radiometric instruments are of the utmost value, and these methods are indispensable for energy measurements, but for mapping spectral detail there is no question that photography, where it can be applied, is far superior both in refinement of observation and in ease of manipulation.

From general considerations, therefore, it would seem to be important to extend the application of photography toward the infra-red, as well as toward the ultra-violet.

\section{HISTORICAL}

Iodern commercial photographic plates are comparatively insensitive beyond about $\lambda_{5}$. For longer wave lengths specially prepared emulsions have been used with some success, but in order to obtain any considerable extension into the red there has been more activity of recent years, in sensitizing ordinary dry plates by means of certain dyes. The literature on this sub- 
ject has become so extensive that it can not be reviewed here. ${ }^{5}$ The dyes which have been most used in astronomical spectroscopy seem to be erythrosin, pinacyanol, and a combination of homocol, pinaverdol, and pinacyanol recommended by Wallace. ${ }^{6}$ By the use of pinacyanol, stellar spectra have been quite widely observed in the $\mathrm{H} \alpha\left(\lambda 6_{563}\right)$ region, and have been pushed as far as $\lambda_{7} \mathrm{I}$, but are poorly observed at this extreme wave length because of the rapid decrease in sensitivity. Dicyanin has been tried by several astronomers, ${ }^{7}$ but except in a few instances the limit has not been greatly extended beyond $\lambda_{7}$ I. Dr. Slipher has kindly furnished photographic prints of the originals of the illustrations of stellar spectra in the Encyclopedia Brittannica. These spectra extend well into the red; the $A$ line appears in several, being most distinctly shown in $\alpha$ Orionis.

In view of the favorable results at wave lengths greater than 7I obtained at the Bureau of Standards with dicyanin, it seemed that the full value of the dye had never been realized in astrophysics, and that further trials might be profitable. In I9I2 Dr. Burns had expressed the opinion ${ }^{8}$ that with existing instruments it would be possible to photograph the spectra of bright stars as far as $\lambda 80$.

Lack of complete success heretofore can probably be traced to one or more of the following causes: (a) Deterioration of the dye; (b) failure of the staining process employed to give sufficient sensitivity; or $(c)$ ignorance of the spectral region in which dicyanin is most valuable.

(a) It is an unfortunate fact that dicyanin can not be depended upon to preserve its useful properties indefinitely under ordinary conditions; some chemical change takes place which destroys wholly or in part its sensitizing value. It appears quite probable that numerous tests of dicyanin have been made using samples which were no longer in good condition. The deterioration can be retarded and perhaps nearly eliminated by proper precautions.

(b) The sensitivity of the dyed plate depends on the composition of the staining bath and on the details of the manipulation. Various processes have been recommended by the makers of the

5 Eder and Valenta, Beiträge Zur Photochemie und Spectralanalyse, Wien, I904; Wallace, Astrophysical Journal, 26, p. 31r, rgo7; Baly, Spectroscopy, Chap. XI, London, rgr2; at1d Eder, Photographsche Korrespondenz, September, 1915.

6 Astrophysical Journal, 26, p. 3 xr; 1907.

${ }^{7}$ V. M. Slipher, Astrophysical Journal, 28 ,p. 400, 1908; Enc. Brit., rith ed., 21, p. 717, rgro-11; Bosler, Comptes Rendus, 160, p. 124, r9r5.

8 Publications of the Astronomical Society of the Pacific, 24, p. 25 r; 1912. 
dye and others; some of these processes probably would not give sufficient speed for exposures on faint sources.

(c) Some experimenters have possibly not realized at what wave lengths dicyanin has its greatest value, namely, those greater than 68. Pinacyanol is superior in speed up to about $\lambda 68$, but for continuous and absorption spectra, dicyanin is probably to be preferred even at this point on account of its flatter sensitivity curve.

\section{PURPOSE OF THIS INVESTIGATION.}

The general aim of the investigation reported upon in this paper was to ascertain whether stellar spectra are sufficiently intense in the region of wave length $0.80 \mu(8000 \mathrm{~A})$ to allow these wave lengths to be photographed with moderate exposure times by means of dicyanin. If this proved to be the case, it was hoped that the observations might indicate something of the value of this part of the spectrum to astronomers and physicists.

\section{OBSERVATIONS}

\section{THE INSTRUMENT}

By an arrangement with Director Pickering, of the Harvard College Observatory, the 24 -inch reflector of the observatory was used

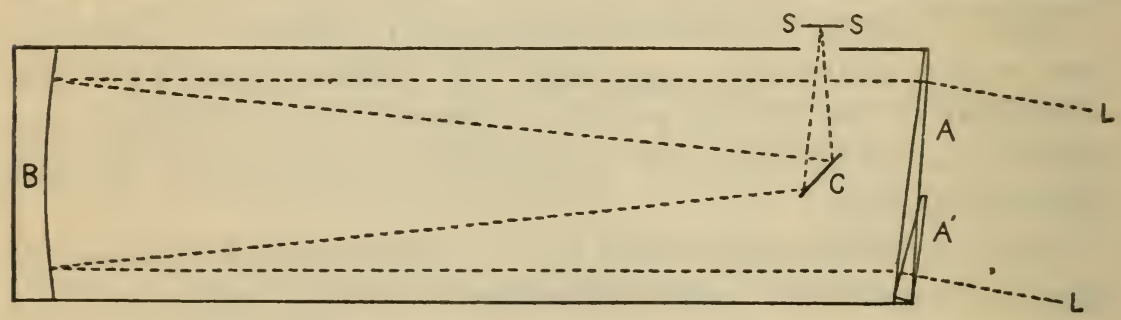

FIG. I.-Schematic diagram of optical system of 24 -inch reflector with objective prism

$L, L$, Light rays from star; $A$, prism $\mathrm{P}$, refracting angle $6^{\circ}$, a perture 24 inches (circular); or $A^{1}$, prism $\mathrm{D}$, refracting angle $15^{\circ}$, aperture 9 by $I I$ inches; $B$, concave mirror, aperture 24 inches, focal length $I I$ feet; $C$, plane mirror; $S-S$, spectrum.

during the month of February, I9I7, to make the tests referred to in the above paragraph. The reflector is of the Newtonian type, with an aperture of 24 inches and a focal length of I f feet. The spectra were formed by an objective prism placed at the upper end of the tube above the secondary mirror. This arrangement, due to Prof. Pickering, combining as it does great light-gathering power with achromatism, was obviously well suited to the problem in hand. Two prisms were used: Prism P, circular, aperture 24 inches, angle about $6^{\circ}$; Prism D, nearly rectangular, 9 by I I inches, angle about $15^{\circ}$. The latter prism was placed eccentrically 
at the mouth of the tube in order that the secondary mirror might not occult too much of the light Thus, practically the whole aperture of the prism was made use of. (See Fig. r.) The linear dispersions yielded by the prisms are as follows:

Linear Dispersion

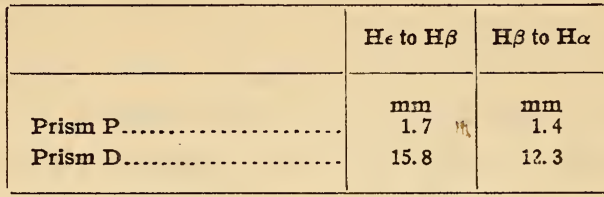

The telescope is equipped with electric drive and pendulum control which afforded great convenience in obtaining any desired departure from sidereal rate.

\section{PHOTOGRAPHIC PROCESSES}

The exact action of the dye in sensitizing plates is so little understood, and the subject is so complex, that in spite of the large amount of research upon it, any statements made at this time in regard to the best procedure must be regarded as provisional, as they are more than likely to be revised by subsequent experience. Similarly, certain precautions mentioned below may later be shown to be unnecessary.

Both dicyanin and a more recent product, dicyanin $\mathrm{A}$, were tried at Harvard. The difference in the action of the two dyes is not very marked, dicyanin A, perhaps, being better for the very long waves. The dyes should be obtained as soon after manufacture as possible and should be kept cool and free from moisture. The stock solution should be made in the ratio I : rooo or I : 2000 in the highest grade of absolute ethyl alcohol. The best results obtained by the writer have been with the following procedure:

- Staining Solution

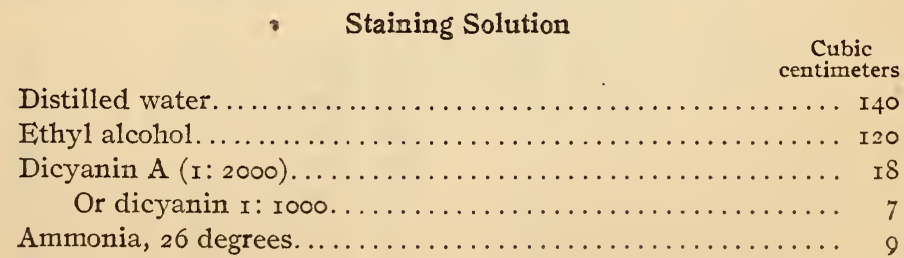

Directions for Sensitizing. - Mix the water and alcohol thoroughly and allow to stand. After adding the dye allow several minutes before the ammonia is put in and again an interval before staining. Bathe the plates $4 \frac{\mathrm{T}}{2}$ minutes at $20^{\circ} \mathrm{C}\left(68^{\circ} \mathrm{F}\right)$. Place for about 40 seconds in a bath of pure alcohol at the same temperature, remove, 
and dry in a current of air. Avoid high temperatures at any point of the process.

The bath can be used repeatedly if 3 or $4 \mathrm{c} \mathrm{c}$ of ammonia is added each time. The dye and all solutions should be protected from light. In packing the plates after staining do not allow the film to come in contact with any solid object, even glass or another film. The stained plates deteriorate rather quickly; if placed in an ice box, they will keep fairly well for a few days, but it is advisable to sensitize them as shortly before using as convenient. Use any clear working developer ${ }^{10}$ a few degrees cooler than usual.

The fact that dicyanin does not sensitize the plates to green and yellow light is made apparent by the appearance of the photographed spectra. As the illustrations show, the blue and red portions of the spectra are usually separated by a gap representing the insensitive region. A more nearly uniform spectrum can undoubtedly be obtained by the addition of pinaverdol ${ }^{11}$ to the staining bath.

\section{SPECTROSCOPIC RESULTS}

Between February 4 and March I, I9I7, 67 stellar spectrograms were secured on 14 nights. Those of interest are included in the following list: Columns one to seven require no explanation. In the eighth column, "Exposure," c indicates that the observation was interfered with by clouds; in the ninth column, "Prism," P denotes the 24 -inch $6^{\circ}$ prism; D denotes the 9 by II inch $15^{\circ}$ prism; in the tenth column, "Emulsion," HS indicates Hammer Special, 27 indicates Seed 27 ; in the last column, "Dye," D and DA signify dicyanin and dicyanin A, respectively.

Journal of Observations

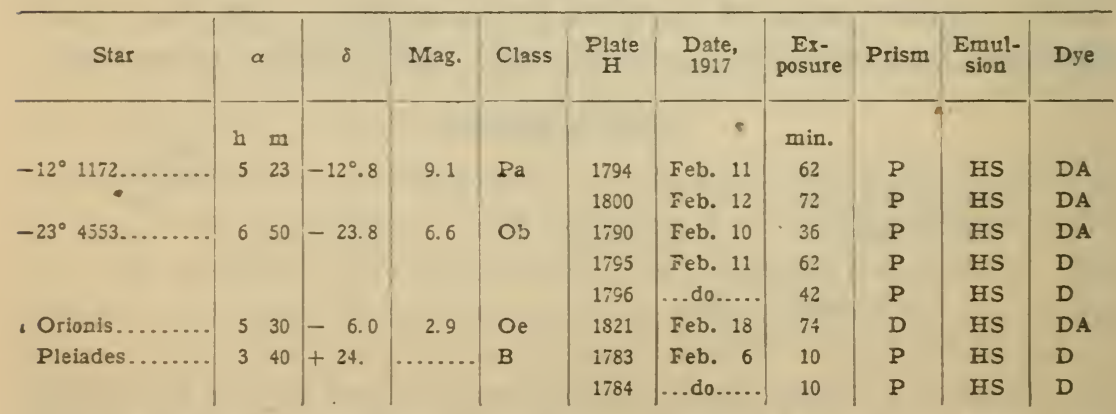

\footnotetext{
9 The above procedure is much the same as that recommended by Wallace for his three-dye stain, Astrophysical Journal, 26, p. $31 x$; 1907.

${ }^{10}$ In this investigation paramidophenol (I part to 16 parts of water), as recommended by Prof. King, was used and seemed satisfactory for the dicyanin plates.

12 Possibly also erythrosin or orthochrome $T$ or other dy'es could be used, but they have not been actually tried in combination with dicyanin by the writer. Dicyanin and pinaverdol have been used together many times at the Bureau of Standards in photographing laboratory spectra.
} 
Journal of Observations-Continued

\begin{tabular}{|c|c|c|c|c|c|c|c|c|c|c|c|}
\hline Star & & $\alpha$ & $\delta$ & Mag. & Class & $\begin{array}{l}\text { Plate } \\
\text { H }\end{array}$ & $\begin{array}{l}\text { Date, } \\
1917\end{array}$ & $\begin{array}{c}\text { Ex- } \\
\text { posure }\end{array}$ & Prism & $\begin{array}{c}\text { Emul- } \\
\text { sion }\end{array}$ & Dye \\
\hline$\beta$ Orionis. & $\begin{array}{l}\mathrm{h} \\
5\end{array}$ & $\begin{array}{l}\mathbf{m} \\
10\end{array}$ & $-8^{\circ} .3$ & 0.3 & B8 & 1787 & Feb. 10 & $\frac{\min .}{14}$ & $P$ & HS & D \\
\hline$\kappa$ Draconis. & 12 & 29 & +70.3 & 3.9 & B5P & 1813 & Feb. 16 & $54 c$ & D & 27 & DA \\
\hline \multirow[t]{2}{*}{ 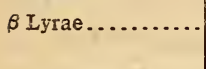 } & 18 & 46 & +33.2 & ( ) & $\mathrm{Bp}$ & 1829 & Feb. 18 & $25 c$ & D & HS & $\mathrm{D}$ \\
\hline & & & & & & 1844 & Feb. 24 & 38 & D & HS & DA \\
\hline P Cygni...... & 20 & 15 & +37.7 & 4.9 & B1p & 1845 & ...do..... & $20 \mathrm{c}$ & D & HS & DA \\
\hline \multirow{4}{*}{$\alpha$ Can. Maj........ } & 6 & 41 & -16.6 & -1.6 & Ao & 1791 & Feb. 10 & 16 & D & HS & D \\
\hline & & & & & & 1810 & Feb. 16 & $42 c$ & $P$ & 27 & DA \\
\hline & & & & & & 1822 & Feb. 18 & 39 & $\mathbf{P}$ & HS & DA \\
\hline & & & & & & 1839 & Feb. 24 & 60 & $P$ & HS & $\mathrm{D}$ \\
\hline$\alpha$ Boötis... & 14 & 11 & +19.7 & 0.2 & $\mathrm{~K}_{0}$ & 1815 & Feb. 16 & $56 \mathrm{c}$ & P & EIS & DA \\
\hline$\alpha$ Urs. Maj... & 10 & 58 & +62.3 & 2.0 & $\mathrm{~K}_{0}$ & 1825 & Feb. 18 & 37 & $\mathbf{P}$ & HS & DA \\
\hline \multirow[t]{2}{*}{$\alpha$ Tauri............. } & 4 & 30 & +16.3 & 1.1 & $\mathrm{~K} 5$ & 1785 & Feb. 6 & 5 & D & HS & $D$ \\
\hline & & & & & & 1807 & Feb. 14 & $35 \mathrm{c}$ & $\mathbf{P}$ & HS & DA \\
\hline \multirow[t]{3}{*}{$\alpha$ Orionis.. } & 5 & 50 & +7.4 & 0.9 & $\mathrm{Ma}$ & 1789 & Feb. 10 & 17 & $P$ & HS & $\mathrm{DA}$ \\
\hline & & & & & & 1809 & Feb. 16 & $24 \mathrm{c}$ & D & 27 & DA \\
\hline & & & & & & 1832 & Feb. 21 & 75 & D & HS & D \\
\hline \multirow[t]{2}{*}{$\lambda$ Draconis.. } & 11 & 25 & +69.9 & 4.1 & $\mathrm{Ma}$ & 1793 & Feb. 10 & $24 \mathrm{c}$ & $\mathbf{P}$ & HS & DA \\
\hline & & & & & & 1834 & Feb. 21 & 65 & D & HS & D \\
\hline \multirow[t]{5}{*}{$\alpha$ Herculis.. } & 17 & 10 & +14.5 & 3.5 & $\mathbf{M b}$ & 1817 & Feb. 16 & 7 & D & HS & DA \\
\hline & & & & & & 1828 & Feb. 18 & $38 \mathrm{c}$ & $D$ & HS & D \\
\hline & & & & & & 1837 & Feb. 22 & $22 \mathrm{c}$ & D & HS & D \\
\hline & & & & & & 1843 & Feb. 24 & 52 & D & HS & DA \\
\hline & & & & & & 1849 & Feb. 28 & $23 \mathrm{c}$ & $\mathbf{P}$ & 27 & DA \\
\hline$\psi$ Virginis..... & 12 & 51 & -9.0 & 4.9 & $\mathbf{M}(\mathrm{b})$ & 1814 & Feb. 16 & $66 \mathrm{c}$ & D & 27 & DA \\
\hline \multirow[t]{2}{*}{ HR $5589 . \ldots \ldots \ldots$} & 14 & 56 & +66.3 & 4.9 & $\mathrm{Mb}$ & 1842 & Feb. 24 & 30 & D & HS & DA \\
\hline & & & & & & 1847 & Feb. 28 & $41 \mathrm{c}$ & $\mathbf{P}$ & 27 & DA \\
\hline 40 Com. Ber.. & 13 & 2 & +23.2 & 5.9 & $\mathrm{Mbp}$ & 1841 & Feb. 24 & 72 & D & FS & DA \\
\hline \multirow[t]{2}{*}{ - Ceti................ } & 2 & 14 & -3.4 & $(5.7)$ & Md9 & 1831 & Feb. 21 & 68 & D & HS & D \\
\hline & & & & (5.8) & & 1838 & Feb. 24 & 72 & $D$ & HS & D \\
\hline \multirow[t]{4}{*}{ R Leo. Min......... } & 9 & 39 & +35.0 & $(7.8)$ & Md8 & 1797 & Feb. 11 & 32 & $P$ & HS & D \\
\hline & & & & $(7.8)$ & ......... & 1804 & Feb. 12 & 62 & $\mathrm{P}$ & HS & DA \\
\hline & & & & (7.9) & & 1824 & Feb. 18 & 43 & $D$ & Fis & D \\
\hline & & & & $(7.9)$ & & 1830 & Feb. 20 & $112 \mathrm{c}$ & D & HS & $D$ \\
\hline \multirow[t]{3}{*}{ U Hydrae........... } & 10 & 33 & -12.8 & (5.4) & $\mathbf{N}$ & 1792 & Feb. 10 & $22 \mathrm{c} ?$ & $\mathbf{P}$ & HS & D \\
\hline & & & & (5.4) & & 1798 & Feb, 11 & 24 & P & HS & $D$ \\
\hline & & & & (5.4) & . & 1812 & Feb. 16 & $50 c$ & D & 27 & DA \\
\hline \multirow[t]{3}{*}{$\mathrm{DM}+38^{\circ} 1539 \ldots}$. & 6 & 30 & +38.5 & 6.3 & N & 1802 & Feb. 12 & 71 & $P$ & HS & DA \\
\hline & & & & & & 1811 & Feb. 16 & $49 \mathrm{c}$ & D & 27 & DA \\
\hline & & & & & & 1833 & Feb. 21 & 72 & $D$ & HS & $D$ \\
\hline \multirow[t]{2}{*}{ Y Hydrae.......... } & 9 & 46 & -22.6 & (6.7) & $I_{p}$ & 1840 & Feb. 24 & $78 c ?$ & D & HS & DA \\
\hline & & & & (6.8) & & $18 \div 6$ & Feb. 28 & $46 c$ & $\mathbf{P}$ & 27 & DA \\
\hline $\mathrm{DM}+61^{\circ} 667 \ldots$ & 3 & 57 & +61.5 & 7.9 & $\mathrm{~N}$ & 1799 & Feb. 12 & 16 & $\mathbf{P}$ & HS & DA \\
\hline $\mathrm{DM}+14^{\circ} 1283 \ldots$ & 6 & 20 & +14.8 & 6.5 & $\mathrm{~N}$ & 1801 & ...do...... & 72 & $\mathrm{P}$ & HS & DA \\
\hline $\mathrm{DI}+46^{\circ} 817 \ldots$ & 12 & 40 & +46.0 & ( ) & N & 1827 & Feb. 18 & $54 c$ & D & HS & D \\
\hline $\mathrm{DM}+68^{\circ} 617 \ldots$ & 10 & 38 & +67.9 & ( ) & $\mathrm{N}$ & 1826 & ...do..... & 70 & D & HS & $\mathrm{D}$ \\
\hline $\mathrm{DM}-24^{\circ} 12084 \ldots .$. & 15 & 22 & -24.8 & 7.4 & $\mathbf{R}$ & 1805 & Feb. 12 & 16 & $P$ & HS & DA \\
\hline $\mathrm{DM}+42^{\circ} 2811 \ldots \ldots$ & 17 & 13 & +42.2 & 7.3 & $\mathbf{R}$ & 1848 & Feb. 28 & $41 \mathrm{c}$ & $\mathrm{P}$ & HS & DA \\
\hline
\end{tabular}

Description of the spectra obtained will now be taken up in the order of the Harvard classification. ${ }^{12}$ Wave lengths were deter-

${ }^{12}$ Annals Harvard College Observatory, 2S, p. I40; 56, p. 66. 
mined or identified by micrometer measurement of the photographs. Those given in italics are the results as measured. The accuracy of the various measurements is indicated by the number of figures given in stating the wave lengths. The units will be omitted, since it will be understood that all wave lengths referred to lie between 3000 and 10000 angstroms ( 0.3 and $1.0 \mu)$. The accuracy attained is not great, and the plates probably fail to show many of the finer details of the spectra. This brief study is therefore in the nature of a reconnoissance. The results indicate the desirability of future observations in the extreme red and infrared regions with both objective-prism and slit spectroscopes.

$D M-I 2^{\circ}$ II72-Spectral Class Pa.-In the spectrum of this interesting planetary nebula the following emission lines, all previously observed, are shown: 3728 (nebular), $\mathrm{H} \delta, \mathrm{H} \gamma, \mathrm{H} \beta$, $\mathrm{H} \alpha . \mathrm{H} \alpha$ is the strongest line, 3728 next. There is a suggestion of an emission line of somewhat shorter wave length than $\mathrm{H} \alpha$, and on one plate a faint trace of an emission line at about $7 \mathrm{I} 4$. $\mathrm{H} \alpha$ has been observed visually by Campbell ${ }^{13}$ and photographically by Bosler. ${ }^{14}$ Campbell remarked that $\mathrm{H} \alpha$ was "very difficult." This is in striking contrast with my photographs on which $\mathrm{H} \alpha$ is the strongest line of all.

$D M-23^{\circ} 4553 .-$ Emission lines only were observed in this Wolf-Rayet star as follows:

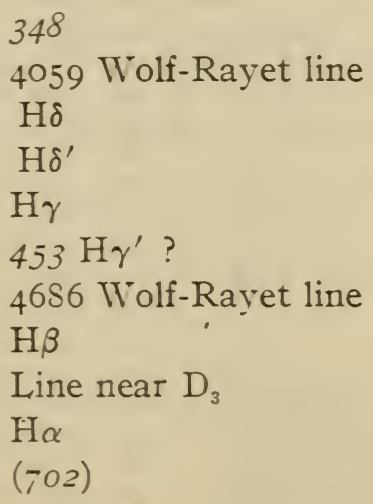

The italicized numbers above, as also those shown on succeeding pages of this paper, are wave lengths as measured. The identifications assumed for the other lines depend also upon measurements. The strongest line in the spectrum is $4686 ; 348$ is a distinct, well-marked line on two plates. It may have the 
same origin as a line in about this position observed by Palmer ${ }^{15}$ in Nova Persei and in two planetary nebulae N. G. C. 674I and N. G. C. 6886, but the identification is doubtful. The wave lengths of 702 from different plates are not accordant, but the line looks real. Lines at 69I and 7II have been observed by Bosler ${ }^{16}$ in other Wolf-Rayet stars. He did not observe this star. $\mathrm{H} \alpha$ was the only line in the red seen by Campbell. ${ }^{17}$

-Orionis-Spectral Class Oe.-The terrestrial atmospheric absorption lines $B, a$, and $A$ are the only details observed in the red. $\mathrm{H} \alpha$ is lost by overexposure. In the microscope of power io the spectrum can be seen to about 796 . With the naked eye it can be traced, perhaps, 200 angstroms farther. Unless otherwise specified, the limits mentioned for other stars refer to the appearance of the spectrum in the microscope. With dicyanin the spectrum shades off gradually, so that these limits are indefinite, but care has been taken not to overestimate them.

Stars of Class B.-On account of the limited time at my disposal only a few spectra of the earlier classes were obtained. In Class $B$ no new features were discovered. The characteristic intensity curve is well shown by the plate of the Pleiades, Fig. 6. It is interesting to compare the relative intensities of the two portions of the spectrum with the intensities shown by stars of other classes. The differences are so great that the class could be determined fairly well from an image so weak or so poorly defined that no lines are visible.

Bright-Line Spectra of Class B.-Several spectra of this type were photographed, including $\kappa$ Draconis, $\beta$ Lyrae, and PCygni. $\mathrm{H} \alpha$ is a strong emission line in all these spectra. The photographs of $\beta$ Lyrae and of P Cygni were taken under poor conditions and hence are not so valuable as they might otherwise be. Besides $\mathrm{D}_{3}$ and $\mathrm{H} \alpha$ several emission lines of greater wave length are seen with low power. These probably include 6678 and 7065 of helium. The spectra extend to about 75 .

aCanis Majoris-Spectral Class.Ao.-The plate of low dispersion is overexposed in the stronger portions of the spectrum. The following features have been measured in the less refrangible part: 627 , slight maximum in continuous spectrum; 650, minimum in continuous spectrum; 693 , center of strong broad maximum; 727 , drop in intensity; $87-89$, end.

\footnotetext{
15 Lick Observatory Bulletins, 2, p. 46; 1902.

16 Comptes Rendus, 160, p. I24; I9I5.

17 Astronomy and Astrophysics, 8, p. 456; rS94.
} 
The longer spectra show the absorption lines $\mathrm{H} \alpha, B, a, A . \quad A$ is strong and conspicuous. There is a decided suggestion of an absorption line at about $\mathcal{S}_{1}$. The spectrum extends faintly to 84 . (See Fig. 2.)

$\alpha$ Boötis-Spectral Class Ko.-The features measured include $K, H, g$, absorption band head at $5166, A$, band head (?) at 808 , end of spectrum 854 .

$\alpha$ Ursae Majoris-Spectral Class Ko.-This spectrum, which is not so strongly exposed as that of $\alpha$ Boötis, shows $K, H, g$, band head at.5166, D, B, A. In both spectra there is apparently some faint detail beyond $A$, but it is too poorly shown to be interpreted.

$\alpha$ Tauri-Spectral. Class $K_{5}$. - The spectrum taken with the $6^{\circ}$ prism is too short to allow many features in the red to be distinguished. There is a broad maximum at 70 , while the spectrum ends about 82. An absorption line (probably $A$ ) beyond the maximum is seen with low power. (See Fig. 7.)

The longer spectrum, which is not strongly exposed in the red, shows $D, H \alpha, B, a, A$. There is a broad maximum of the continuous spectrum between $B$ and $a$.

$\alpha$ Orionis-Spectral Class Ma.-On the small dispersion plate $K, H, g, A$, and a slight maximum at 79 , were measured in addition to heads of absorption bands at $4760,4955,5166,5862,6156$, and 7054. These bands all head toward shorter wave lengths, and are well known in Class M spectra. The spectrum extends to 85 .

The following features were measured in the longer spectra: $K, H, g, D, B, A$, and band heads at $4955,5166,6156,7054$. The end of the spectrum as measured on the two plates falls at 833 and 874. (See Fig. 3.)

On all the plates of $\alpha$ Orionis there is apparently an absorption band with its head (toward the violet) nearly coincident with $A$. It probably represents the early stage of a band which develops to great prominence in $\mathrm{Mb}$ and $\mathrm{Md}$ spectra. In common with many other bands in the spectrum of $\alpha$ Orionis, this new band is almost undoubtedly to be ascribed to titanium oxide. The identification will be discussed later in this paper.

$\lambda$ Draconis-Spectral Class Ma.-The bands are not so pronounced as in $\alpha$ Orionis. $A$ is well shown on the high dispersion plate, but the band having its head at that point is not in evidence.

$\alpha$ Herculis-Spectral Class $M b$. - This star has a spectrum which is rendered very striking by the strong flutings found throughout a long range of wave lengths. The plate of small dispersion shows 


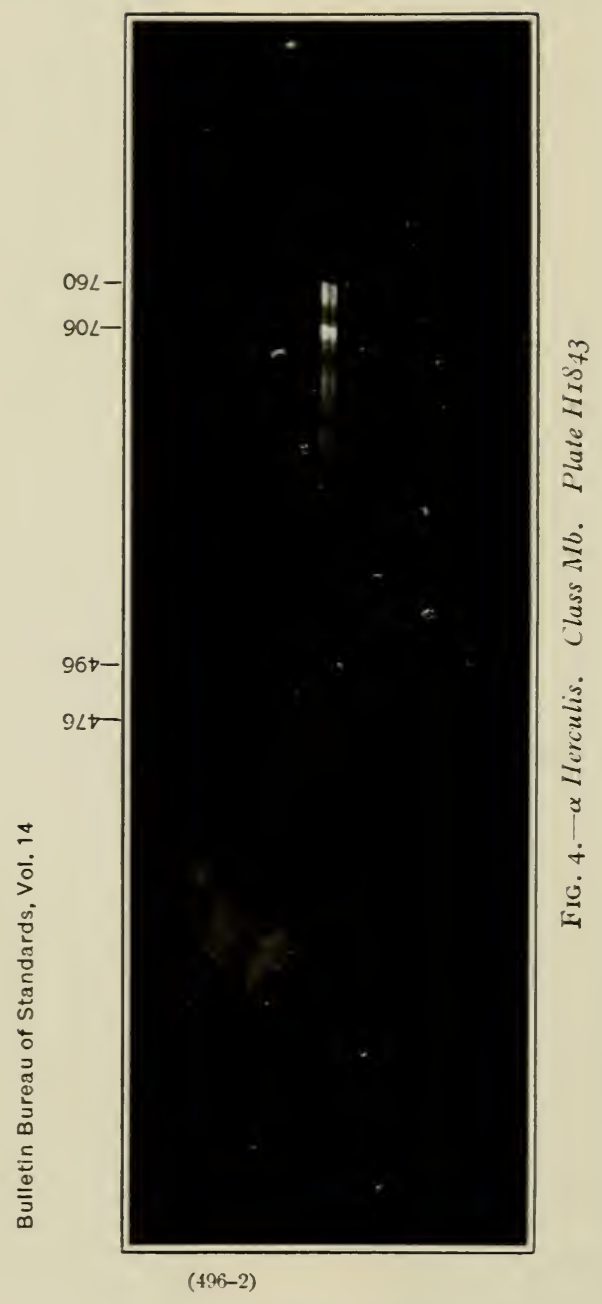


the following features: $K, H, g, A$, and band heads at 4760,4955 , $5166,5862,6156,6652,706,760$. There is a distinct maximum at 808 , perhaps indicating an absorption band beginning at a somewhat greater wave length. The spectrum is observed to 83 .

With higher dispersion the spectrum contains so much detail that an adequate description is difficult. The chief features are shown in Fig. 4. The measurements include settings on $g, B, A$, and band heads at $476,4955,5763,5815,585,6$ I $56,706,760$.

On the best plate the band head at 706 is distinctly triple. Assuming the first component to lie at 7054, the wave lengths of the second and third were measured as 709 and 713 . These are well-known titanium oxide bands observed in the laboratory by Fowler, ${ }^{18}$ and by Hale and Adams ${ }^{19}$ in both laboratory and sunspot spectra. Referring to stellar spectra, these bands have been observed in $\alpha$ Orionis by Newall and Cookson, ${ }^{20}$ and by Slipher. ${ }^{21}$ The latter observed them, partially, in the spectrum of o Ceti. ${ }^{22}$ On two plates there is seen what may be the head of a rather weak absorption band at $8 I I$. This agrees with the appearance of the short spectrum as described above. The settings on the end of the spectrum range on different plates from 825 to 836 . A faint continuation is visible without magnification to a considerably greater wave length. As this is true in the spectra of other stars also, it is worth noting because it indicates that the sensitivity curve of the dicyanin plate does not drop abruptly, and that greater effective exposures will record stellar. spectra to even longer wave lengths than those obtained in this investigation.

The absorption band beginning sharply near $A$ and shading off toward longer waves is strongly deleped, and with the fluting at 706 gives the extreme red portion of the spectrum a remarkable appearance. The spectrum here might conveniently be described as consisting of two emission bands sharply cut off at 706 and 760 , and gradually weakening toward the violet. As these features of the spectrum seem to vary considerably from star to star, it is not improbable that they may be useful in distinguishing the subdivisions of Class M. The band of greater wave length seems to become intensified with advance in spectral type.

$\psi$ Virginis-Spectral Class $M(b)$.- Although classified as Mb this spectrum resembles that of $\lambda$ Draconis more than that of $\alpha$ Herculis. It does not appear to be more advanced than $\alpha$ Orionis. A single

21 Monthly Notices, R. A. S., 69, p. $510 ;$
22 Astrophysical Joumal, 25, p. 236; 1907. 
spectrum of $\psi$ Virginis was secured and its quality is only fair, but the appearance of the bands in the red favors the classification Ma rather than $\mathrm{Mb}$.

HR 5589-Spectral Class Mb. - This appears to be a typical Mb spectrum. On Plate $\mathrm{H}_{1} \delta_{47}$ (small dispersion) the following features were measured: $K, H, g, A$, band heads at $4760,4955,5$ r 66 , $586,61_{5} 6,6652,706,76$. The spectrum ends more abruptly than usual at $\mathcal{S}_{3}$. (See Fig. 8.)

40 Come Berenicis-Spectral Class Mb.-This spectrum is similar to HR 55 S.

o Ceti-Spectral Class Md 9.-The characteristics distinguishing Class Mb from Class Ma are still more pronounced in this Md spectrum. The absorption in the flutings is more nearly complete, giving the spectrum a curiously disconnected appearance. By far the most conspicuous features of the whole spectrum, as seen on these plates, Fig. 5, are the two (apparently) emission flutings at 706 and 760 . While these are probably the unshaded portions of continuous spectrum between absorption bands, the maxima are much stronger than anything else seen in the spectrum. The measurements include $B, A$, end of spectrum at 837 , and band heads at $4505,4626,476,4955,5843,6156,656,665 \pm, 706,760$, and possibly one between $\delta_{I}$ and $\delta_{2}$. $\mathrm{H} \delta$ and $\mathrm{H} \gamma$ are seen as emission lines, the former being the stronger.

$R$ Leonis Minoris-Spectral Class $M d$ 8.-In the short spectra the following were observed: $H$ (and $K$ ), emission $H \delta$, trace of emission $\mathrm{H} \gamma$, maximum at $814 \pm$, end 83 , bands at 4760,4955 , 5862,6 r $_{5} 6,6652,706,760$ (reinforced by $A$ ).

In the longer spectra, which are both underexposed, the stronger bands and emission $\mathrm{H} \delta$ are seen. As in o Ceti the portions of continuous spectrum about 69 and about 74 are the most striking features of the spectrum. Bright $\mathrm{H} \delta$ is very strong.

Stars of Class $N$. - The spectra of Class $\mathrm{N}$ as shown on these plates contain considerable detail, but most of it is of such a character that it can not be measured with accuracy. While no precision can be claimed for the wave-length determinations, they are sufficient to identify the main features and to furnish a fairly definite description of what is seen on the photographs. (See Fig. 9.)

In the following tabulations of measurements the terms "rise" and "drop" refer to changes in the intensity curve when proceeding toward longer waves. "pr" indicates present. 
Bulletin Bureau of Standards, Vol. 14

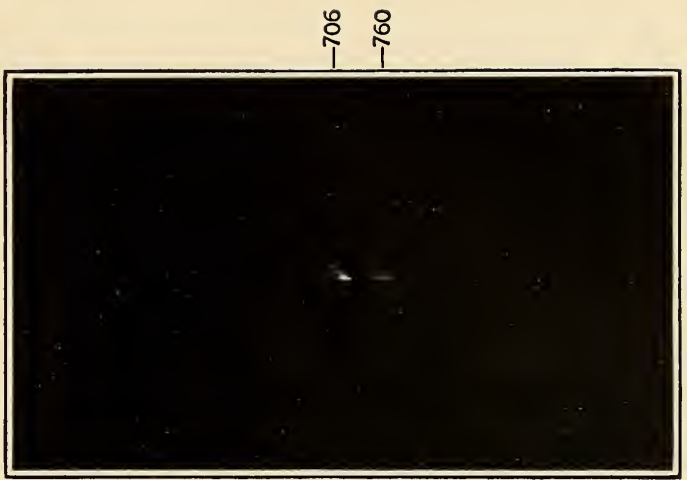

FIg. 5.-o Ceti. Class Mdg. Plate $11 I 83 I$

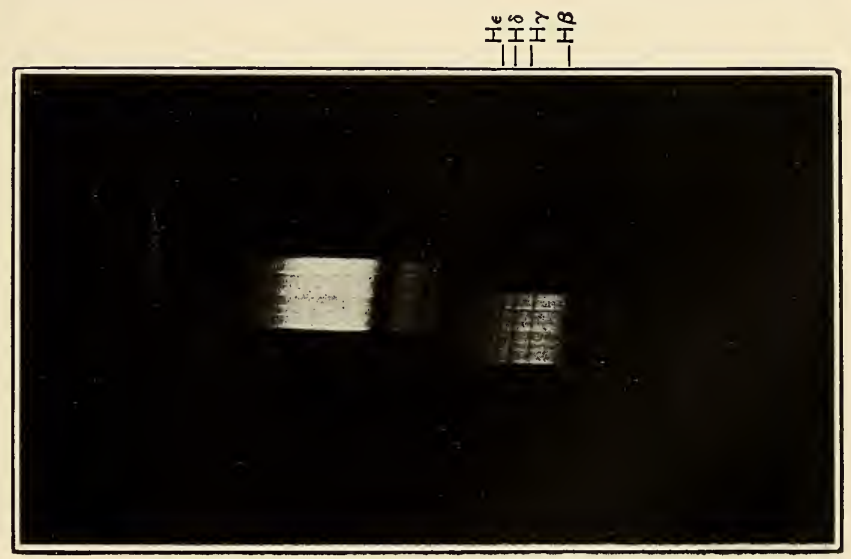

Fig. 6.-Atlas and Pleione. Class B. Plate $\mathrm{HI}_{7} \mathrm{~B}_{3}$ $(498-1)$ 


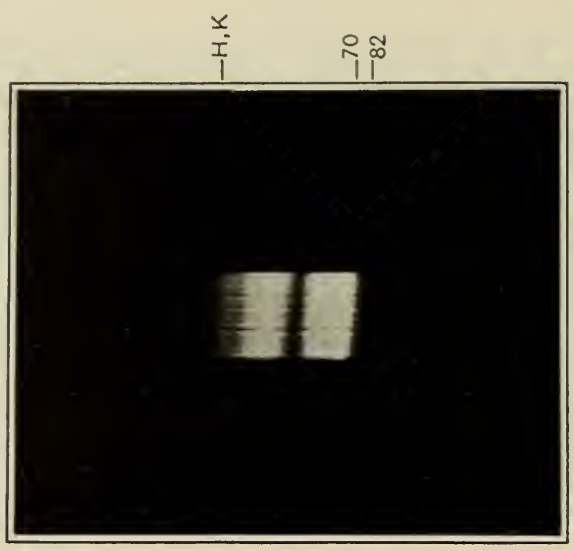

Fig. 7.- $\alpha$ Tauri. Class $K_{5}$. Plate $\mathrm{HI}_{7} \mathrm{~S}_{5}$

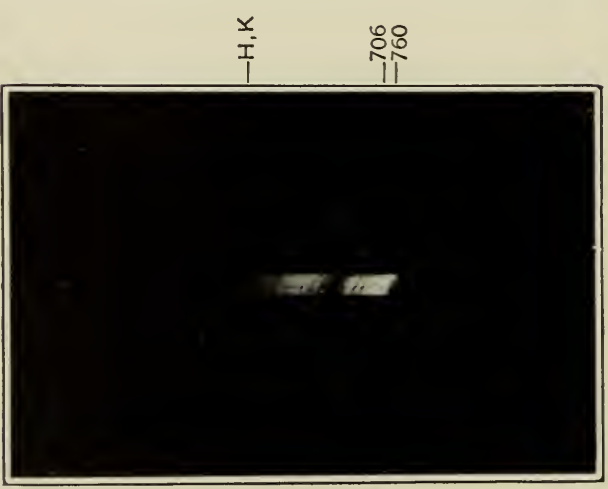

Fig. 8.-H. R. 5589 . Class Mb. Plate HI 447 (499-2) 
Stars of Class $N$

OBSERVATIONS WITH PRISM P

\begin{tabular}{|c|c|c|c|c|c|}
\hline \multirow{2}{*}{ Description } & \multicolumn{2}{|c|}{ U Hydrae } & \multirow{2}{*}{$\begin{array}{c}+38^{\circ} 1539, \\
\text { H } 1802\end{array}$} & \multirow{2}{*}{$\begin{array}{c}+14^{\circ} 1283 \\
\mathrm{H} 1801\end{array}$} & \multirow{2}{*}{ Identification } \\
\hline & H 1792 & H 1798 & & & \\
\hline Absorption line........ & 437 & 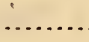 & $436^{5}$ & & Swan band? \\
\hline Absorption $454-474 \ldots .$. & $\mathrm{pr}$ & $\mathrm{pr}$ & $\mathrm{pr}$ & $\mathrm{pr}$ & Swan band \\
\hline Rise................. & 517 & & & & Do \\
\hline Rise $564 \ldots \ldots \ldots \ldots \ldots . . . . . .$. & $\mathrm{pr}$ & pr & $\mathrm{pr}$ & $\mathrm{pr}$ & Do \\
\hline Absorption line $591 \ldots \ldots$. & $\mathrm{pr}$ & pr & $\mathrm{pr}$ & $\mathrm{pr}$ & Cyanogen band? \\
\hline Broad maximum......... & 681 & 683 & 682 & 683 & \\
\hline Absorption line? ............ & ......... & 729 & 731 & .......... & Do \\
\hline Narrow absorption line..... & & & 758 & & Probably A \\
\hline 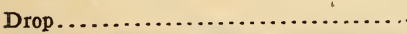 & & & 786 & & \\
\hline Narrow absorption line? ........... & & & $(802)$ & & \\
\hline Absorption line? .................... & $(90)$ & (91) & $(90)$ & & \\
\hline
\end{tabular}

OBSERVATIONS WITH PRISM D

\begin{tabular}{|c|c|c|c|c|c|}
\hline \multirow{2}{*}{ Description } & \multirow{2}{*}{$\begin{array}{c}\text { U Hydrae, } \\
\text { H } 1812\end{array}$} & \multicolumn{2}{|c|}{$\mathrm{D} M+38^{\circ} 1539$} & \multirow{2}{*}{$\begin{array}{c}\text { DM+46 } \\
817, \mathrm{H} 1827\end{array}$} & \multirow{2}{*}{ Identification } \\
\hline & & H 1811 & H 1833 & & \\
\hline Rise.... & t & & 5597 & & Detached portion \\
\hline Do............ & 5643 & & 5645 & 5630 & Swan band \\
\hline Drop........ & ....... & $\ldots$ & 5730 & … & Cyanogen band? \\
\hline Rise.............. & ......... & 5805 & 5810 & ...... & \\
\hline Absorption line ......... & 5876 & ........ & 5909 & ..... & Do \\
\hline Maximum............ & ..... & $\ldots$ & $68 \%$ & 687 & \\
\hline Drop..................... & $69 \mathrm{r}$ & 691 & $693^{5}$ & 692 & Do \\
\hline Do..................... & 707 & 708 & $709^{8}$ & 707 & Do \\
\hline Do.................... & 722 & 724 & & $\ldots$ & Do \\
\hline A................... & $\mathrm{pr}$ & $\mathrm{pr}$ & $\mathrm{pr}$ & ........ & \\
\hline End....... & $79+$ & & $82 I$ & 795 & \\
\hline
\end{tabular}

Low dispersion-Prism P.-At 682 there is a strong broad maximum in the continuous spectrum. It rises so abruptly on the side of shorter wave length, at about 67 , as to give the impression that an absorption band terminates here, but the higher dispersion does not bear out that interpretation. This maximum may arise from a combination of the intensity curve of the star and of the dispersion of the prism, which condenses a considerable amount of energy in a comparatively narrow space. The change with wave length of the sensitivity of the emulsion of course adds its effect. These considerations will be briefly discussed later in this paper.

The absorption line measured at 73 , as shown by the longer spectra, is in reality an absorption band with its head at 72 . 
Beyond so the spectra are very weak-in fact almost invisiblebut an indication of an absorption line at a great wave length, approximately 90 , was measured on three plates. The reality of this line on any one plate is so doubtful that the measurements would not be recorded here except for the circumstance that all three yield the same wave length as closely as could be expected in the case of an actual line. This, however, does not prove the existence of the line (or band), which must be left to future observations to decide. If the line is real, it should be clearly shown by effective exposures two or three times as long as those received by these plates. Such exposures are not out of the question.

The stars $\mathrm{Y} \mathrm{Hydrae}$ and $\mathrm{D} \mathrm{M}+6 \mathrm{I}^{\circ} 667$ appear with small dispersion to be similar to $\mathrm{U}$ Hydrae.

High dispersion-Prism D.-Very little is seen in the ordinary photographic region in these high-dispersion spectra owing to underexposure, and the other portions are not so strong as is desirable. Nevertheless, several new features are clearly indicated. The most important of these are the absorption bands beginning at 692,708 , 723 and degenerating toward the red. They face in the opposite direction from the strong bands of shorter wave length characteristic of Class N. Several of the new features seem to correspond with cyanogen bands as listed by Kayser, ${ }^{23}$ and since other cyanogen bands have previously been found in similar stars ${ }^{21}$ this identification is suggested for them. Unfortunately, this identification is little more than formal, as we are not certain of the origin of these bands. Certain "cyanogen" bands have been observed in the spectrum of a copper arc burning in nitrogen..$^{25}$ The writer is not informed as to whether or not this applies to all of what is ordinarily called the cyanogen spectrum, but we can no longer accept the designation cyanogen at its face value. If we reject the connection between "cyanogen" flutings and a carbon source, it seems a remarkable thing that two sets of bands ordinarily associated with carbon, namely, the Swan spectrum and the cyanogen spectrum should appear together in a stellar source, as they do in the spectrum of a carbon arc. It is impossible, horrever, to press this argument to a conclusion because of incomplete data. The need for further laboratory research upon banded spectra, particu-

\footnotetext{
2 Handbuch der Spectroscapie, 5, p. 228.

24 Hale, Ellerman, and Parikhurst, Publications of the Yerkes Observatory, 2. p. irs; r903.

\$ Uhier and Patterson, Astrophysical Journal, f2, p. 435, I9r5; also Grotian and Runge, Physikalische Zeitschrit, 15, P. 545, 2954 .
} 
larly in the red, is emphasized; and it is not unlikely that stellar spectra of Class $\mathrm{N}$, when more completely observed, will be of value to the laboratory physicist.

$\mathrm{Y}$ Hydrae and $\mathrm{D} \mathrm{M}+68^{\circ} 6 \mathrm{I} 7$, as judged by one underexposed plate of each, possess spectra which seem to be essentially similar to U Hydrae and D M $+38^{\circ}$ I 539 .

Stars of Class R. - Two spectra of Class R, D M-24 ${ }^{\circ}$ I 2084 and $\mathrm{D} \cdot \mathrm{M}+42^{\circ} 28 \mathrm{II}$, were photographed. The plates are not of the best quality, the details not being well shown, but the great departure from Class $\mathrm{N}$ in the matter of spectral distribution of energy is evident at a glance. In the Class $\mathrm{R}$ spectra the blue and red portions are of approximately equal intensity, more or less as in Class $\mathrm{K}$, and decidedly at variance with Class $N$. Taking the central portion of the spectrum, say about 48 , as a standard of comparison, the violet rays are stronger and the red rays weaker than in Class $N$. These observations are in harmony with and extend the Harvard descriptions of Class $\mathrm{R}$ and support in an interesting way the analogy between the sequences $G, K, M$, and $G, R, N$, as set forth by Dr. Rufus. ${ }^{26}$ The spectral distribution of intensity in stars of various classes will be further discussed later in this paper.

\section{GENERAL DISCUSSION}

\section{IDENTIFICATION OF BAND AT WAVE LENGTH $.760 \mu$}

As previously described, an absorption band with its head at $\lambda_{7} 60$, about coincident with Fraunhofer $A$, and extending toward longer wave lengths, has been observed in spectra belonging to various subdivisions of class $M$. This band appears to increase in strength with advancing spectral type. Since it occurs in spectra in which other bands, also heading toward shorter wave lengths, are known to be due to titanium oxide, it was natural to suppose that this might have the same origin. There are several published descriptions and illustrations of the titanium oxide spectrum, but none that the writer has seen refers to wave lengths as great as 760. Hence this portion of the spectrum was photographed by him since returning to Washington. The arc formed between electrodes of metallic titanium was projected upon the slit of the spectroscope. The arc burned brilliantly at 2 or 3 amperes, but displayed an annoying tendency to wander about over the elec-

${ }^{20}$ Publications of the Astronomical Observatory of the University of Michigan, 2, p. ro3; rgr5. $110990^{\circ}-19-2$ 
trodes. ${ }^{27}$ Since the banded spectrum is stronger in the outer envelope of the arc an attempt was made to observe this part of it, but the position of the image of the arc upon the slit could not be satisfactorily controlled for reasons just referred to. Nevertheless, photographs were obtained in which the flutings stand out quite prominently, and as was anticipated, a band at about $\lambda_{7} 60$ is present. An illustration (Fig. 10) shows this new band as it appears in a one-prism spectroscope. Photographs with a 2 I-foot grating (Fig. I I) show that it is multiple, the first head being near $\lambda 7590$ with others toward longer waves following at intervals of 30 to 40 angstroms, one of the most pronounced being at $\lambda_{7} 66$. Traces of the multiple character are seen on a few of the stellar spectrograms.

\section{DISTRIBUTION OF ENERGY IN STELLAR SPECTRA}

When solar or stellar spectra are photographed with a prism spectroscope on red sensitive plates there is usually found to be a decided maximum of intensity at some point in the red. With pinacyanol it occurs at $\lambda 66-67$, with dicyanin near $\lambda 70$, but this does not necessarily imply real maxima either in the stellar spectra or in the sensitizing power of the dyes. The opacity of the photographic emulsion at different points in a spectrum depends upon many factors, the three most important being (I) spectral intensity of the source (energy per angstrom); (2) dispersion of the spectroscope (angstroms per millimeter); and (3) sensitivity of the photographic emulsion (darkening per unit of incident energy). Neglecting many complications, the ordinates of the opacity curve are found by multiplying together the corresponding ordinates of the curves (I), (2), and (3). Now, even though the intensity and the sensitivity steadily decrease toward the red, it may happen that at a certain point the condensation of energy due to the dispersion curve will cause an increase in the photographic action, while a little farther along the spectrum this is overbalanced again by smaller values of intensity and sensitivity. These considerations may explain certain maxima so commonly shown in stellar spectra, particularly in the red. If so, no great importance is to be attached to them.

While actual spectral-energy curves can not be drawn from the data at hand, the great differences between those of various types

${ }^{27}$ Bars or rods of metallic titanium were not obtainable at this time, hence it was necessary to use pieces of irregular shape. 
Bulletin Bureau of Standards, Vol. 14

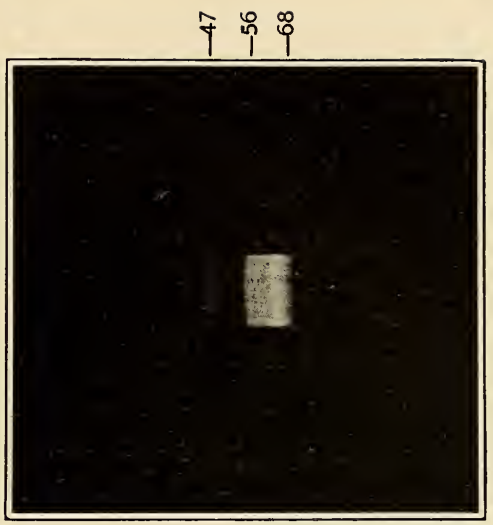

Fig. 9. $-D M+38^{\circ}$ 1539. Class N. Plate HI802

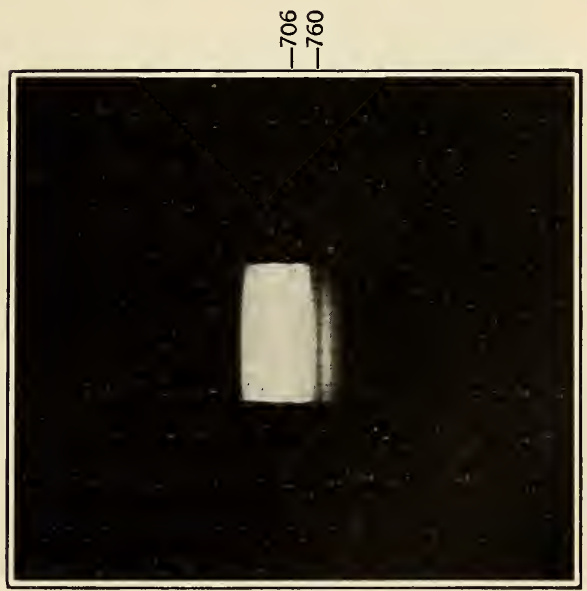

FIG. Io.-Titanium arc; one-prism spectrograph (502-1) 


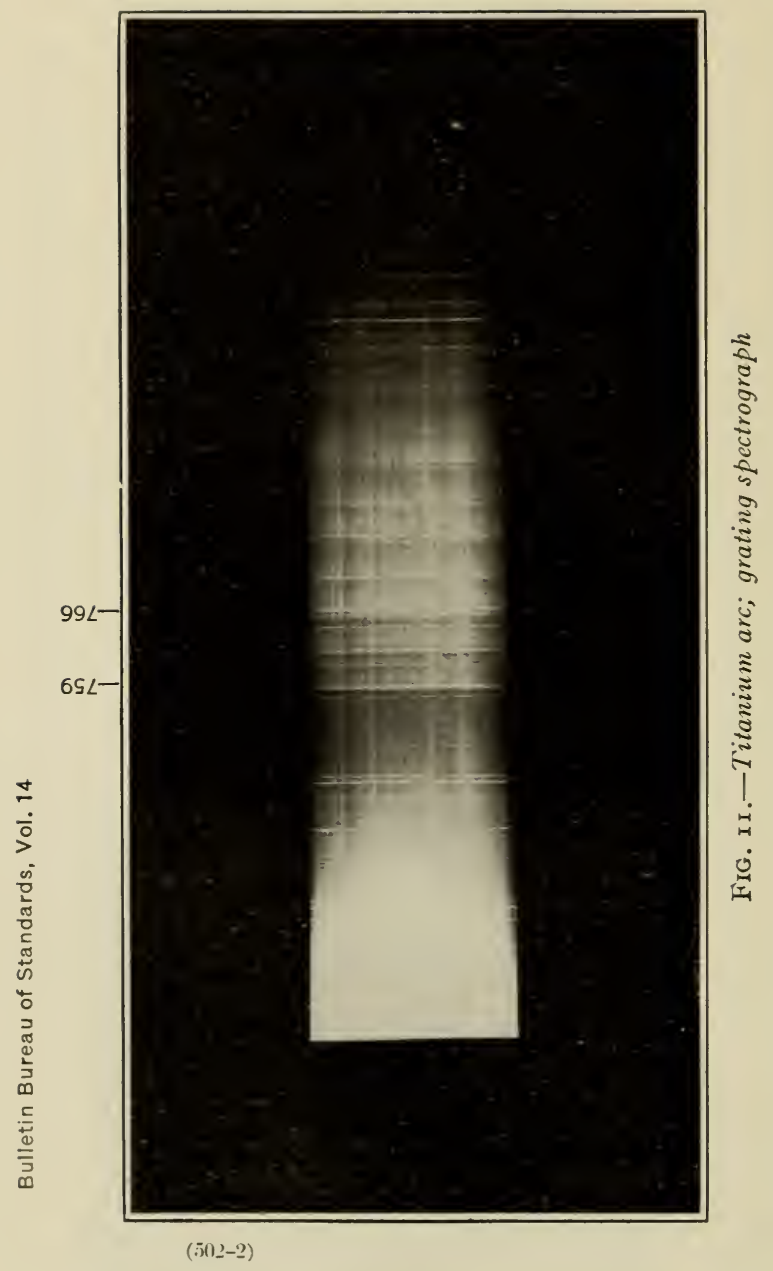


of stars have been emphasized by the photographs taken during this investigation.

Nearly all stellar spectra taken with moderate exposures on dicyanin plates appear in two parts, there being a gap in the yellow-green, due to the insensitiveness of the emulsion. It is instructive to observe the steady shift of energy from the blue to the red side of this gap in passing from early to late types of spectra. For classes $B$ and $A$ the blue portion is much the stronger; at class $\mathrm{K}$ the two parts are about equal; while for classes $\mathrm{M}$ and $\mathrm{N}$ the red portion is stronger. The great contrast in energy distribution in the different classes is clearly shown in the illustrations. It has been determined, roughly, in a numerical way by estimating the relative amounts of photographic action in the two portions of nearly Ioo spectra appearing on the plates of small dispersion. The table shows the ratio of the red to the blue portion. At my request Dr. Burns made independent estimates of the same stars. The two series agree well where the ratio is near unity, and in other cases do not differ more than might be expected from the uncertainties in estimates of this kind.

Ratio of Red to Blue Light-Average Values

\begin{tabular}{|c|c|c|}
\hline Class & Burns & Merrill \\
\hline $\mathbf{B}, \mathbf{A} \ldots \ldots$ & 0.1 & 0.2 \\
\hline$F, G \ldots \ldots$. & .4 & .5 \\
\hline K.......................... & 1.2 & 1.2 \\
\hline $\mathbf{M}$ & 1.3 & 1.3 \\
\hline $\mathrm{N} \ldots \ldots \ldots \ldots$ & 4.1 & 3.6 \\
\hline R....................... & .9 & .8 \\
\hline
\end{tabular}

It is somewhat surprising to find so small a difference between Classes $\mathrm{K}$ and $\mathrm{M}$. This does not necessarily contradict the belief that stars of Class $\mathrm{M}$ are considerably redder than those of Class $\mathrm{K}$, for these spectra refer in part to waves too long to affect the eye, and it is probably at great wave lengths that the strong absorption bands of titanium oxide partly compensate the increasing redness.

The ratio $B$ and $A$ to $N$ is $4 \mathrm{I}$ according to Burns's estimates and 18 according to the writer's, ${ }^{28}$ corresponding to magnitude differences of 4.0 and 3.I, respectively. This would undoubtedly be considerably increased if we could compare wave lengths shorter than 40 with those longer than 65 .

${ }^{28}$ Burns's estimates are evidently on a more extended scale than the writer's and probably more nearly correspond to the actual energy ratios. 
Only two Class $\mathrm{R}$ stars are included, but the results are of special interest. The estimates are as follows:

Ratio of Red to Blue Light-Class $\mathbf{R}$

\begin{tabular}{|c|c|c|}
\hline & Buras & Mersill \\
\hline$-24^{\circ} 1258 \%$. & 0.55 & 2. 0 \\
\hline$+42^{\circ} 2811 \ldots$ & .35 & .7 \\
\hline Mean & so &.$\varepsilon \Xi$ \\
\hline
\end{tabular}

These numbers show in a definite way the wide divergence from Class $\mathrm{N}$ and the similarity to Class $\mathrm{K}$, as referred to previously in this article. The correspondence of these two stars is apparently to an early rather than to a late subdivision of Class $\mathrm{K}$.

\section{SUMMARY}

I. Fraunhofer's A band (wave length $0.760 \mu$ ) and a considerable region of greater wave length have been photographed in numerous stellar spectra by means of plates sensitized with dicyanin.

2. A strong band at $0.760 \mu$, nearly coincident with $A$, has been discovered in spectra of Class $M$. It is very marked in $\mathrm{Mb}$ and Md spectra, and may be useful for purposes of classification.

3. The titanium arc shows flutings near this wave length, which probably correspond to the stellar bands.

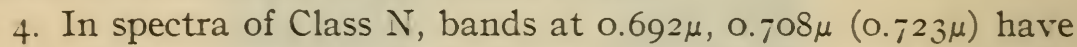
been observed, which may be identical in origin with bands in the so-called cyanogen spectrum.

5. On dicyanin plates, spectra of Class $\mathrm{R}$ are strikingly dissimilar to those of Class $\mathrm{N}$ in spectral distribution of energy.

6. The greatest wave lengths observed appear by extrapolation to be about $0.87 \mu$.

7. General conclusions may be stated as follows: (a) Many stellar spectra possess sufficient intensity in the region of wave length $0.80 \mu$ (infra-red) to enable this portion of the spectrum to be photographed on plates sensitized with dicyanin; $(b)$ in farorable instances stellar spectra can be well observed to $0 . \$_{5} \mu$, or possibly to even greater wave lengths; $(c)$ the region of stellar spectra of greater wave length than $0.70 \mu$ contains features of importance, especially in the case of the later types. 
Acknowledgment is hereby made of the courtesy of Director Pickering, of the Harvard College Observatory, in making this investigation possible, and of the aid rendered by him, by Prof. King, Miss Cannon, and other members of the staff during the progress of the observations.

WASHINGTON, April I2, I9I 7 . 